\title{
Unimodular Lattices for the Gaussian Wiretap Channel
}

\author{
Jean-Claude Belfiore and Patrick Solé \\ Telecom ParisTech, LTCI UMR 5141 \\ 46, rue Barrault \\ 75013 Paris, France \\ Email: \{jean-claude.belfiore, patrick.sole\}@ telecom-paristech.fr
}

\begin{abstract}
In [1] the authors introduced a lattice invariant called "Secrecy Gain" which measures the confusion experienced by a passive eavesdropper on the Gaussian Wiretap Channel. We study, here, the behavior of this invariant for unimodular lattices by using tools from Modular Forms and show that, for some families of unimodular lattices, indexed by the dimension, the secrecy gain exponentially goes to infinity with the dimension.
\end{abstract}

\section{INTRODUCTION}

The wiretap channel was introduced by Wyner [2] as a discrete memoryless broadcast channel where the sender, Alice, transmits confidential messages to a legal receiver Bob, in the presence of an eavesdropper Eve. Wyner defined the perfect secrecy capacity as the maximum amount of information that Alice can send to Bob while insuring that Eve gets a negligeable amount of information. He also described a generic coding strategy known as coset coding. While coset coding has been used in many coding scenarios (for ex. [3], [4]), Wyner used it to encode both data and random bits to confuse the eavesdropper. The question of determining the secrecy capacity of many classes of channels has been addressed extensively recently, yielding a plethora of information theoretical results on secrecy capacity.

There is a sharp contrast with the situation of wiretap code designs, where very little is known. The most exploited approach to get practical codes so far has been to use LDPC codes (for example [5] for binary erasure and symmetric channels, [6] for Gaussian channels with binary inputs). We also note that wiretap II codes have been extended to more general settings such as network coding in [7]. Finally, lattice codes for Gaussian channels have been considered from an information theoretical point of view in [8].

In [1], a design criterion for constructing explicit lattice codes, has been proposed, based on the analysis of Eve's correct decision probability. This design criterion relies on a new lattice invariant called "secrecy gain" based on theta series. In this paper, we analyze the secrecy gain for unimodular lattices.

The paper is organized as follows. In Section [II] we recall the definition of the secrecy gain and give its value for some extremal even unimodular lattices. An asymptotic analysis is then performed in section IV for even unimodular lattices and we prove that the secrecy gains of some of these lattices grow up to infinity with the dimension. Finally we come back to the Gaussian wiretap channel in section $\square$ to show that unimodular lattices only define one operating point for the system. Some other types of lattices should be studied in the future for other operating points.

\section{NOTATIONS AND PREVIOUS RESULTS}

\section{A. Notations and system model}

We use, in this paper, the same system model and the same notations as [1]. In [1], the secrecy gain has been defined and some examples have been given. Here we analyze more deeply this parameter for even unimodular lattices and give the asymptotic behavior of this secrecy gain when the dimension of the lattices grows to infinity. Figure 1 gives the model

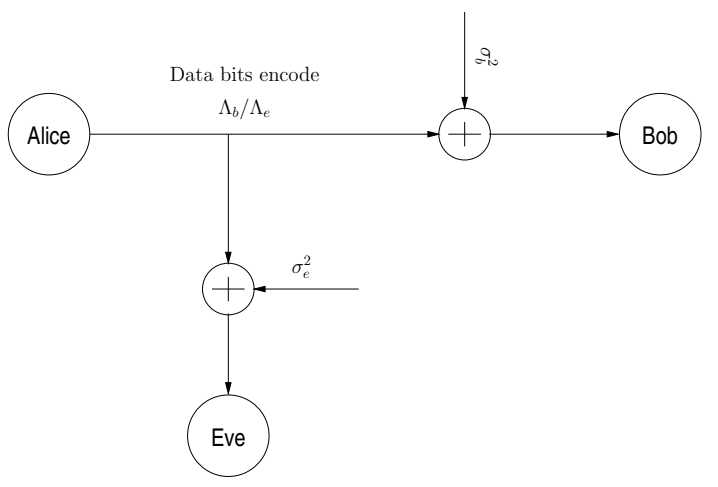

Figure 1. The Gaussian Wiretap Channel

considered in this paper where Alice wants to send data to Bob on a Gaussian channel whose noise variance is given by $\sigma_{b}^{2}$. Eve is the eavesdropper trying to intercept data through another Gaussian channel whose noise variance is $\sigma_{e}^{2}$. In order to have a positive secrecy capacity, we will assume that $\sigma_{e}^{2}>\sigma_{b}^{2}$. Bits are transmitted by Alice at a rate equal to $R=R_{s}+R_{r}$ where $R_{s}$ is the secrecy rate of this transmission and $R_{r}$ is the rate of pseudo-random bits. Indeed, we use Wyner's generic coding strategy [9]. We give the remaining parameters,

- $\Lambda_{b}$ is the fine lattice (used to minimize Bob's probability of error)

- $\Lambda_{e}$ is the coarse lattice (used to minimize Eve's probability of correct decision)

- $n$ is the dimension of both lattices 
- $\mathcal{V}\left(\Lambda_{b}\right)$ (resp. $\mathcal{V}\left(\Lambda_{e}\right)$ ) is the fundamental parallelotope of $\Lambda_{b}\left(\right.$ resp. $\left.\Lambda_{e}\right)$

- $\operatorname{Vol}(\mathcal{P})$ is the volume of $\mathcal{P}$

Data bits label cosets in $\Lambda_{b} / \Lambda_{e}$ while pseudo-random bits label points of $\Lambda_{e}$. The reader can refer to [1] for a more detailed description of the coding scheme. Still according to [1], and under the assumption of a moderate to high secrecy rate, the expression of the probability of correct decision at the eavesdropper can be expressed as

$$
P_{c, e} \simeq\left(\frac{1}{\sqrt{2 \pi} \sigma_{e}}\right)^{n} \operatorname{Vol}\left(\mathcal{V}\left(\Lambda_{b}\right)\right) \sum_{\mathbf{r} \in \Lambda_{e}} e^{-\frac{\|\mathbf{r}\|^{2}}{2 \sigma_{e}^{2}}} .
$$

In eq. (1), we recognize the theta series of lattice $\Lambda_{e}$.

\section{B. Theta series of a lattice}

Definition 1: Let $\Lambda$ be a Euclidean lattice, then the theta series of $\Lambda$ is [10]

$$
\Theta_{\Lambda}(z) \triangleq \sum_{\boldsymbol{x} \in \Lambda} q^{\|\boldsymbol{x}\|^{2}}, q=e^{i \pi z}, \operatorname{Im}(z)>0
$$

Some exceptional lattices have theta series that can be expressed as functions of the Jacobi theta functions $\vartheta_{i}(q)$, $i=2,3,4$ with

$$
\begin{aligned}
& \vartheta_{2}(q)=\sum_{n=-\infty}^{+\infty} q^{\left(n+\frac{1}{2}\right)^{2}} \\
& \vartheta_{3}(q)=\sum_{n=-\infty}^{+\infty} q^{n^{2}} \\
& \vartheta_{4}(q)=\sum_{n=-\infty}^{+\infty}(-1)^{n} q^{n^{2}}
\end{aligned}
$$

For instance, table $\square$ gives the theta series of some exceptional lattices.

\begin{tabular}{|c|c|}
\hline Lattice $\Lambda$ & Theta series $\Theta_{\Lambda}$ \\
\hline \hline Cubic lattice $\mathbb{Z}^{n}$ & $\vartheta_{3}^{n}$ \\
\hline$D_{n}$ & $\frac{1}{2}\left(\vartheta_{3}^{n}+\vartheta_{4}^{n}\right)$ \\
\hline Gosset lattice $E_{8}$ & $\frac{1}{2}\left(\vartheta_{2}^{8}+\vartheta_{3}^{8}+\vartheta_{4}^{8}\right)$ \\
\hline \multicolumn{2}{|c|}{ Table I }
\end{tabular}

THETA SERIES OF SOME LATTICES

\section{Minimization of the theta series}

One problem that arises naturally when studying theta series is the following. In eq. (1), set $y=i z$ and restrict to real values of $y$. We are now interested in studying

$$
\Theta_{\Lambda}(y)=\sum_{\boldsymbol{x} \in \Lambda} q^{\|\boldsymbol{x}\|^{2}}, q=e^{-\pi y}, y>0 .
$$

Equation (1), giving Eve's probability of correct decision, can be written as

$$
P_{c, e} \simeq\left(\frac{1}{\sqrt{2 \pi} \sigma_{e}}\right)^{n} \operatorname{Vol}\left(\mathcal{V}\left(\Lambda_{b}\right)\right) \Theta_{\Lambda_{e}}\left(\frac{1}{2 \pi \sigma_{e}^{2}}\right)
$$

So, for a given dimension $n$, the problem to solve is to find a lattice $\Lambda^{\text {opt }}$ that minimizes $\Theta_{\Lambda}(y)$ for a given value of $y$ in order to minimize expression (3).

\section{THE SECRECY GAIN}

\section{A. Definitions}

We recall here some definitions given in [1].

We remark that, if we do not use any specific coarse lattice $\Lambda_{e}$, we can assume that $\Lambda_{e}$ is equal to a scaled version of $\mathbb{Z}^{n}$ with same volume as $\Lambda_{e}$. Consequently, for a lattice $\Lambda$, it is natural to define the secrecy function. For a lattice with unitary volume, we have

Definition 2: Let $\Lambda$ be an $n$-dimensional lattice with unitary volume. The secrecy function of $\Lambda$ is

$$
\Xi_{\Lambda}(y) \triangleq \frac{\Theta_{\mathbb{Z}^{n}}(y)}{\Theta_{\Lambda}(y)}=\frac{\vartheta_{3}(y)^{n}}{\Theta_{\Lambda}(y)}
$$

defined for $y>0$.

Then of course, as we want to minimize the expression of Eve's probability of correct decision in eq. (3), we are interested in the maximum value of the secrecy function. So, we define the secrecy gain,

Definition 3: The secrecy gain of an $n$-dimensional lattice $\Lambda$ is

$$
\chi_{\Lambda} \triangleq \sup _{y>0} \Xi_{\Lambda}(y)
$$

\section{B. The secrecy gain of unimodular lattices}

Theta series are difficult to analyze. Nevertheless, for some lattices, these functions have nice properties. It is the case of even unimodular lattices whose theta series are modular forms with integer weight. We mainly restrict this paper to the study of even unimodular lattices and will use tools from modular forms.

1) Definitions and formulas: We recall the definition of an integral lattice [10],

Definition 4: A lattice $\Lambda$ is integral if its Gram matrix has entries in $\mathbb{Z}$. Note that an integral lattice has the property

$$
\Lambda \subseteq \Lambda^{\star} \subseteq \frac{1}{\operatorname{Vol}(\mathcal{V}(\Lambda))^{2}} \Lambda
$$

From this definition, we can now define unimodular lattices,

Definition 5: A lattice $\Lambda$ is unimodular if

1) $\Lambda$ is integral

2) $\Lambda$ is equal to its dual

Note that a unimodular lattice has fundamental volume equal to 1 .

Let $\Lambda^{\star}$ be the dual lattice of the $n$-dimensional lattice $\Lambda$. Then Jacobi's formula [10] gives the theta series of $\Lambda^{\star}$ as a function of the theta series of $\Lambda$,

$$
\Theta_{\Lambda^{\star}}(y)=\operatorname{Vol}(\mathcal{V}(\Lambda)) y^{-\frac{n}{2}} \Theta_{\Lambda}\left(\frac{1}{y}\right)
$$

If $\Lambda$ is unimodular, then using (4), we deduce

$$
\Theta_{\Lambda}(y)=\Theta_{\Lambda^{\star}}(y)=y^{-\frac{n}{2}} \Theta_{\Lambda}\left(\frac{1}{y}\right) .
$$

So, since $\mathbb{Z}^{n}$ itself is unimodular, the secrecy function of $\Lambda$ has the property,

$$
\Xi_{\Lambda}(y)=\Xi_{\Lambda}\left(\frac{1}{y}\right)
$$


If we express $y$ in decibel (in our case, $y=\frac{1}{2 \pi \sigma^{2}}$ and is related to Eve's signal to noise ratio), then the secrecy function becomes an even function.

Conjecture 1: The secrecy gain of unimodular lattices is achieved by the secrecy function at $y=1$.

Using conjecture 1 in what follows, we can evaluate the secrecy gain of unimodular lattices as

$$
\chi_{\Lambda}=\Xi_{\Lambda}(1)
$$

Some formulas: Some formulas are useful to calculate the secrecy gain of unimodular lattices. The most important ones, found in [11], are

$$
\begin{aligned}
& \vartheta_{2}\left(e^{-\pi}\right)=\vartheta_{4}\left(e^{-\pi}\right) \\
& \vartheta_{3}\left(e^{-\pi}\right)=\sqrt[4]{2} \vartheta_{4}\left(e^{-\pi}\right)
\end{aligned}
$$

2) Secrecy gain of some exceptional unimodular lattices:

a) Gosset Lattice $E_{8}: E_{8}$ is unimodular even. From table I and eq. (5), we get

$$
\begin{aligned}
\frac{1}{\Xi_{E_{8}}(1)} & =\frac{\frac{1}{2}\left(\vartheta_{2}\left(e^{-\pi}\right)^{8}+\vartheta_{3}\left(e^{-\pi}\right)^{8}+\vartheta_{4}\left(e^{-\pi}\right)^{8}\right)}{\vartheta_{3}\left(e^{-\pi}\right)^{8}} \\
& =\frac{1}{2}\left(1+\frac{1}{4}+\frac{1}{4}\right) \\
& =\frac{3}{4}
\end{aligned}
$$

We deduce, then, the secrecy gain of $E_{8}$,

$$
\chi_{E_{8}}=\Xi_{E_{8}}(1)=\frac{4}{3}=1.33333
$$

As an illustration, figure 2 gives the secrecy function of $E_{8}$.

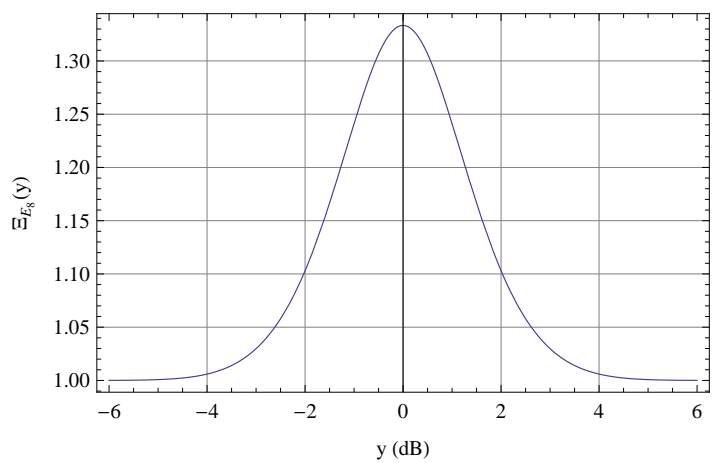

Figure 2. Secrecy function of $E_{8}$

b) Leech Lattice $\Lambda_{24}: \Lambda_{24}$ is also unimodular even. From table I] we get (with simplified notations)

$$
\begin{aligned}
\frac{1}{\Xi_{\Lambda_{24}}(1)} & =\frac{\frac{1}{8}\left(\vartheta_{2}^{8}+\vartheta_{3}^{8}+\vartheta_{4}^{8}\right)^{3}-\frac{45}{16} \vartheta_{2}^{8} \vartheta_{3}^{8} \vartheta_{4}^{8}}{\vartheta_{3}^{24}} \\
& =\frac{27}{2^{6}}-\frac{45}{2^{8}} \\
& =\frac{63}{256}
\end{aligned}
$$

We deduce, then, the secrecy gain of $\Lambda_{24}$,

$$
\chi_{\Lambda_{24}}=\Xi_{\Lambda_{24}}(1)=\frac{256}{63}=4.0635
$$

As an illustration, figure 3 gives the secrecy function of $\Lambda_{24}$.

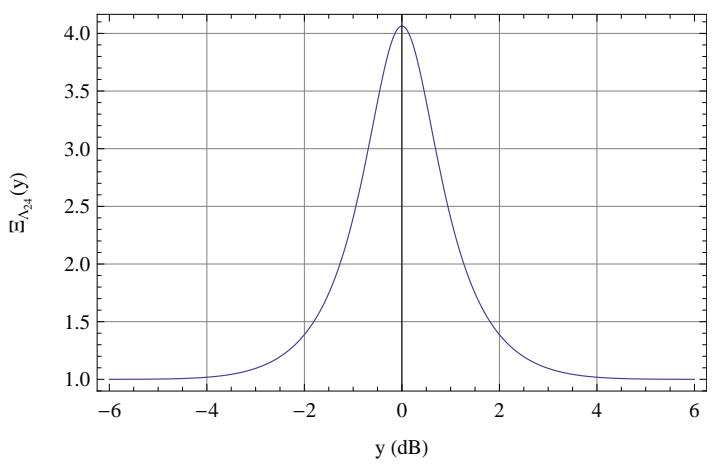

Figure 3. Secrecy function of $\Lambda_{24}$

\section{Higher dimension unimodular extremal lattices}

$E_{8}$ and $\Lambda_{24}$ are extremal even unimodular lattices in dimensions 8 and 24 respectively [10]. Extremal means that their minimum distance is maximal for a given dimension [10]. We can give same type of results for extremal even unimodular lattices of higher dimensions. For instance, we can derive the secrecy functions and secrecy gains of extremal even unimodular lattices in dimensions 32,48 , and 72 using derivations of [12]. The same can be done in dimension 80 by solving a linear system [13]. Please note that, until now, nobody knows if an extremal lattice in dimension 72 exists. Results are summarized in table [II Here we introduce the

\begin{tabular}{|c|c|c|}
\hline Dimension & Lattice $\Lambda$ & $\Theta_{\Lambda}$ \\
\hline \hline 8 & $E_{8}$ & $E_{4}$ \\
\hline 24 & $\Lambda_{24}$ & $E_{4}^{3}-720 \Delta$ \\
\hline 32 & $B W_{32}$ & $E_{4}^{4}-960 E_{4} \Delta$ \\
\hline 48 & $P_{48}$ & $E_{4}^{6}-1440 E_{4}^{3} \Delta+125280 \Delta^{2}$ \\
\hline 72 & $L_{72}$ & $E_{4}^{9}-2160 E_{4}^{6} \Delta+965520 E_{4}^{3} \Delta^{2}-27302400 \Delta^{3}$ \\
\hline 80 & $L_{80}$ & $E_{4}^{10}-2400 E_{4}^{7} \Delta+1360800 E_{4}^{4} \Delta^{2}-103488000 E_{4} \Delta^{3}$ \\
\hline \multicolumn{2}{|c}{ Table II }
\end{tabular}

THETA SERIES OF EXTREMAL LATTICES

function

$$
\Delta(q)=\frac{E_{4}^{3}(q)-E_{6}^{2}(q)}{12^{3}}
$$

where $E_{k}$ are the Eisenstein series [13] defined as

$$
\begin{aligned}
E_{k}(q) & =1+\frac{2}{\zeta(1-k)} \sum_{m=1}^{+\infty} m^{k-1} \frac{q^{m}}{1-q^{m}} \\
& =1-\frac{2 k}{B_{k}} \sum_{m=1}^{+\infty} m^{k-1} \frac{q^{m}}{1-q^{m}}
\end{aligned}
$$

where $B_{k}$ are the Bernouilli numbers [14] and $\zeta(s)$ is the Riemann zeta function

$$
\zeta(s)=\sum_{k=1}^{\infty} \frac{1}{k^{s}} .
$$


Relations with Jacobi functions are (in symbolic notation)

$$
\left\{\begin{array}{l}
E_{4}=\frac{1}{2}\left(\vartheta_{2}^{8}+\vartheta_{3}^{8}+\vartheta_{4}^{8}\right) \\
\Delta=\frac{1}{256} \vartheta_{2}^{8} \vartheta_{3}^{8} \vartheta_{4}^{8}
\end{array}\right.
$$

and give rise to the expressions of theta series evaluated below.

1) Barnes Wall lattice $B W_{32}$ : In dimension 32, BarnesWall lattice $B W_{32}$ is an extremal lattice. We have

$$
\begin{aligned}
\Theta_{B W_{32}}= & \frac{1}{16}\left(\vartheta_{2}^{8}+\vartheta_{3}^{8}+\vartheta_{4}^{8}\right)\left[\left(\vartheta_{2}^{8}+\vartheta_{3}^{8}+\vartheta_{4}^{8}\right)^{3}\right. \\
& \left.-30 \cdot \vartheta_{2}^{8} \cdot \vartheta_{3}^{8} \cdot \vartheta_{4}^{8}\right]
\end{aligned}
$$

so,

$$
\begin{aligned}
\frac{1}{\Xi_{B W_{32}}(1)} & =\frac{1}{16}\left(1+\frac{1}{2}\right)\left[\left(1+\frac{1}{2}\right)^{3}-30 \cdot \frac{1}{16}\right] \\
& =\frac{9}{64} .
\end{aligned}
$$

Hence,

$$
\chi_{B W_{32}}=\frac{64}{9} \simeq 7.11
$$

2) Lattice $P_{48 p(q)}$ : There are two different extremal even unimodular lattices in dimension $48, P_{48 p}$ and $P_{48 q}$ [10, Chap. 5], having, of course the same theta series,

$$
\begin{aligned}
\Theta_{P_{48}}= & \frac{1}{2048}\left[3915 \vartheta_{2}^{16} \vartheta_{3}^{16} \vartheta_{4}^{16}\right. \\
& -1440 \vartheta_{2}^{8} \vartheta_{3}^{8} \vartheta_{4}^{8}\left(\vartheta_{2}^{8}+\vartheta_{3}^{8}+\vartheta_{4}^{8}\right)^{3} \\
& \left.+32\left(\vartheta_{2}^{8}+\vartheta_{3}^{8}+\vartheta_{4}^{8}\right)^{6}\right]
\end{aligned}
$$

giving

$$
\begin{aligned}
\frac{1}{\Xi_{P_{48}}(1)} & =\frac{1}{2048}\left[\frac{3915}{256}-\frac{1440}{16}\left(1+\frac{1}{2}\right)^{3}+32\left(1+\frac{1}{2}\right)^{6}\right] \\
& =\frac{19467}{524288} .
\end{aligned}
$$

Hence,

$$
\chi_{P_{48}}=\frac{524288}{19467} \simeq 26.93
$$

3) Dimensions 72 and 80: In the same way, from table we can compute the secrecy gain for an extremal unimodular even lattice in dimension 72 and 80 . Note that two examples of such lattices in dimension 80 have been given in [15]. We have

$$
\begin{aligned}
& \chi_{\Lambda_{72}}=\frac{134217728}{685881} \simeq 195.69 \\
& \chi_{\Lambda_{80}}=\frac{536870912}{1414413} \simeq 379.57
\end{aligned}
$$

\begin{tabular}{c|c|c|c|c|c|c|}
\hline Dimension & 8 & 24 & 32 & 48 & 72 & 80 \\
\hline \hline Secrecy gain & 1.3 & 4.1 & 7.11 & 26.9 & 195.7 & 380 \\
\hline
\end{tabular}

Table 【II] summarizes all these results.

\section{Asymptotic AnALYsis}

We propose, here to find a lower bound of the best secrecy gain as a function of the dimension $n$, and deduce some asymptotic results (when $n$ is large enough). For a fixed dimension $n$, we compute bounds on the theta series of an optimal unimodular lattice. By optimal, we mean a lattice which maximizes the secrecy gain. We will use the SiegelWeil formula to compute these bounds.

\section{A. A Siegel-Weil formula for theta series of even unimodular lattices}

Let $n \equiv 0(\bmod 8), \Omega_{n}$ be the set of all inequivalent even unimodular $n$-dimensional lattices. Let $k=n / 2$. Then, one has [14]

$$
\sum_{\Lambda \in \Omega_{n}} \frac{\Theta_{\Lambda}(q)}{|\operatorname{Aut}(\Lambda)|}=M_{n} \cdot E_{k}(q)
$$

where

$$
M_{n}=\sum_{\Lambda \in \Omega_{n}} \frac{1}{|\operatorname{Aut}(\Lambda)|}
$$

and $E_{k}(q)$ is the Eisenstein series with weight $k$ even whose expression is given in eq. (6).

Let $\Theta_{\min }^{(n)}=\min _{\Lambda \in \Omega_{n}} \Theta_{\Lambda}$. Then

$$
\Theta_{\min }^{(n)} M_{n} \leq \sum_{\Lambda \in \Omega_{n}} \frac{\Theta_{\Lambda}}{|\operatorname{Aut}(\Lambda)|}=M_{n} E_{k}
$$

giving rise to

$$
\Theta_{\min }^{(n)} \leq E_{k} .
$$

Define

$$
\chi_{n} \triangleq \max _{\Lambda \in \Omega_{n}} \chi_{\Lambda}=\frac{\vartheta_{3}^{n}\left(e^{-\pi}\right)}{\Theta_{\min }^{(n)}\left(e^{-\pi}\right)}
$$

then we get,

$$
\chi_{n} \geq \frac{\vartheta_{3}^{n}\left(e^{-\pi}\right)}{E_{k}\left(e^{-2 \pi}\right)}
$$

\section{B. Limit of $E_{k}$}

Assume $q$ to be a real number $0<q<1$. We have

$$
E_{k}(q)=1+\frac{2 k}{\left|B_{k}\right|} \sum_{m=1}^{+\infty} m^{k-1} \frac{q^{m}}{1-q^{m}}
$$

Replacing $q$ by $e^{-2 \pi}$ gives

$$
E_{k}\left(e^{-2 \pi}\right)=1+\frac{2 k}{\left|B_{k}\right|} \sum_{m=1}^{+\infty} \frac{m^{k-1}}{e^{2 \pi m}-1}
$$

which converges (very quickly) to 2 when $k$ is a multiple of 4 that tends to infinity. Moreover, according to [11], we have

$$
\vartheta_{3}\left(e^{-\pi}\right)=\frac{\pi^{\frac{1}{4}}}{\Gamma\left(\frac{3}{4}\right)} \simeq 1.086>1
$$

so,

$$
\chi_{n} \gtrsim \frac{1}{2}\left(\frac{\pi^{\frac{1}{4}}}{\Gamma\left(\frac{3}{4}\right)}\right)^{n} \simeq \frac{1.086^{n}}{2}
$$



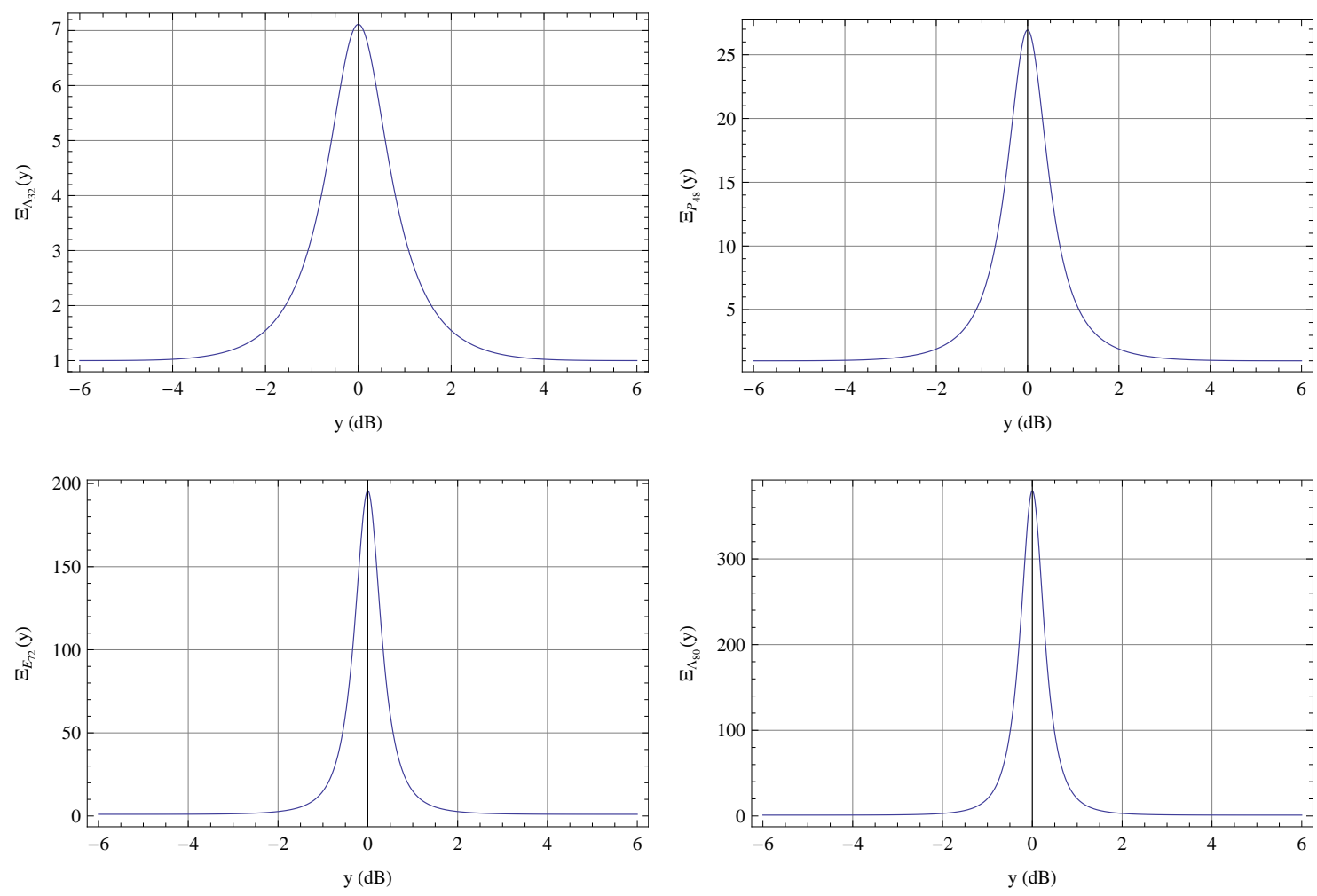

Figure 4. Secrecy functions of extremal lattices in dimensions 32, 48, 72 and 80

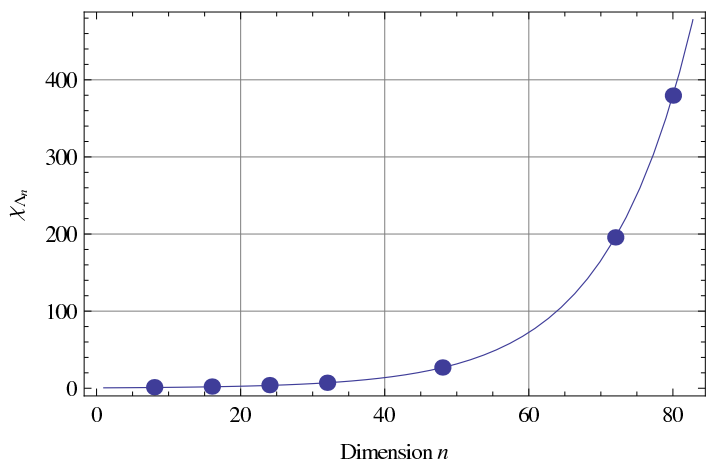

Figure 5. Lower bound of the minimal secrecy gain as a function of $n$ from Siegel-Weil formula. Points correspond to extremal lattices.

which tends exponentially to infinity. Figure 5 gives the asymptotic expression of the secrecy gain as a function of the dimension $n$, as well as points corresponding to extremal lattices in dimensions $8,16,24,32,48,72$ and 80 .

\section{Consequences}

We proved that there exists a family of even unimodular lattices whose secrecy gains exponentially grows up with the dimension, which means that Eve's probability of correct decision exponentially tends to 0 . But as we can remark in figure 4, around its maximum, the secrecy function becomes sharper and sharper when $n$ grows up, which means that, for high dimensions, the communication system absolutely has to operate at $y=1$. We show now, in section $\nabla$, the meaning of this constraint in terms of the communication system as well as the way of doing the same with $y \neq 1$.

\section{BACK TO THE WIRETAP CHANNEL}

We are interested, in this section, in how the secrecy gain is related to the parameters of the Gaussian channel, through the proposed lattice coset construction.

\section{A. Operating point}

For unimodular lattices, the secrecy gain is obtained as the value of the secrecy function at point $y=1$. From eq. (3), it means

$$
\sigma_{e}^{2}=\frac{1}{2 \pi}
$$

Conjecture 1 says that the secrecy gain is achieved by the secrecy function at $y=1$ if the lattice is unimodular. If the lattice is not unimodular, then the secrecy gain can be achieved for another value of $y$. In figure 6, we see that the secrecy gain is achieved for $y=-1.5 \mathrm{~dB}(y=1 / \sqrt{2})$ and not $0 \mathrm{~dB}(y=1)$. We call operating point, the value $y_{\text {o.p. for which }}$

$$
\chi=\Xi\left(y_{\text {o.p. }}\right)
$$

\section{B. Fundamental volume of $\Lambda_{e}$}

The secrecy function is defined for a fundamental volume of $\Lambda_{e}$ equal to 1 . We now relate the secrecy function to system parameters. Let $R$ be the value of the total rate (information bits + pseudo-random bits) transmitted by Alice, per (complex) channel use and $R_{s}$ be the value of the information (secret) rate. 


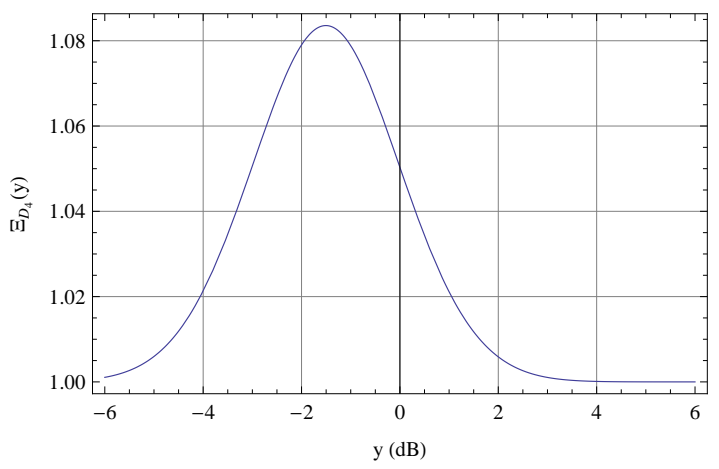

Figure 6. Secrecy function of the checkerboard lattice $D_{4}$

We now establish a correspondence between this parameter $y$ and the physical parameters of the channel.

Fundamental volume of $\Lambda_{e}$ is

$$
\operatorname{Vol}\left(\mathcal{V}\left(\Lambda_{e}\right)\right)=2^{\frac{n R_{s}}{2}} \operatorname{Vol}\left(\mathcal{V}\left(\Lambda_{b}\right)\right)
$$

where $R_{\mathrm{s}}$ is the rate at which Alice sends the secret information bits to Bob. The operating point of the system (for instance volume equal to 1 for a unimodular lattice) corresponds, of course, to a normalized case $y_{\text {norm}}$. In practice, we should work with a scaled lattice. The operating point of this scaled lattice is then,

$$
\begin{aligned}
y & =y_{\text {norm }} \operatorname{Vol}\left(\mathcal{V}\left(\Lambda_{e}\right)\right)^{\frac{2}{n}} \\
& =2^{R_{\mathrm{s}}} y_{\text {norm }} \operatorname{Vol}\left(\mathcal{V}\left(\Lambda_{b}\right)\right)^{\frac{2}{n}}
\end{aligned}
$$

Now, the energy, per channel use, of the signal sent by Alice is

$$
E_{s}=2^{R} \operatorname{Vol}\left(\mathcal{V}\left(\Lambda_{b}\right)\right)^{\frac{2}{n}}
$$

where $R$ is the global rate of the communication (secret bits + pseudo-random bits). Hence, we get

$$
y=2^{R_{\mathrm{s}}} y_{\text {norm }} E_{s} 2^{-R}=2^{-\left(R-R_{\mathrm{s}}\right)} E_{s} y_{\text {norm }} .
$$

As $y_{\text {norm }}=\frac{1}{2 \pi \sigma_{e}^{2}}$, we get

$$
y=\frac{2^{-\left(R-R_{\mathrm{s}}\right)} E_{s}}{2 \pi \sigma_{e}^{2}}=\frac{2^{-\left(R-R_{\mathrm{s}}\right)}}{2 \pi} \gamma_{e}
$$

where $\gamma_{e}$ is Eve's signal to noise ratio. If we use a unimodular lattice, then the operating point is $y=1$ which corresponds to a secrecy rate

$$
R_{s}=R-\log _{2} \frac{2 \pi}{\gamma_{e}}
$$

Other secrecy rates require to study other families of lattices such as modular lattices [16], for instance.

\section{CONCLUSION}

The secrecy gain introduced in [1] is a new lattice invariant that measures how much confusion the eavesdropper will experience. This parameter is based on the value of the theta series of lattice $\Lambda_{e}$ at some point that depends on the lattice itself. We can analyze how secrecy gain behaves, when dimension grows up, if $\Lambda_{e}$ is an even unimodular lattice. In that case, its theta series is a modular form with integer weight and very efficient tools can be used to analyze its behavior. We have shown that an even unimodular lattice with minimal theta series has a secrecy gain which exponentially goes to infinity when dimension $n$ goes to infinity. But this only corresponds to values of Eve's SNR equal to

$$
\gamma_{e}=\pi 2^{R-R_{s}+1}
$$

where $R$ is the total bit rate and $R_{s}$ is the secrecy rate. For other values of $\gamma_{e}$, other families of lattices have to be considered such as $\ell$-modular lattices or their duals. But this requires to find another Siegel-Weil formula for these types of lattices.

\section{REFERENCES}

[1] J.-C. Belfiore and F. Oggier, "Secrecy gain: a wiretap lattice code design," ISITA 2010, 2010. [Online]. Available: arXiv:1004.4075v1[cs.IT]

[2] L. H. Ozarow and A. D. Wyner, "Wire-tap channel II," Bell Syst. Tech. Journal, vol. 63, no. 10, pp. 2135-2157, December 1984.

[3] R. Zamir, S. Shamai, and U. Erez, "Linear/lattice codes for structured multi-terminal binning," IEEE Trans. Inf. Theory, June 2002.

[4] S. Pradhan and K. Ramchandran, "Generalized coset codes for distributed binning," IEEE Trans. Inf. Theory, October 2005.

[5] A. Thangaraj, S. Dihidar, A. R. Calderbank, S. McLaughlin, and J.-M. Merolla, "Applications of LDPC codes to the wiretap channel," IEEE Trans. Inf. Theory, vol. 53, no. 8, August 2007

[6] D.Klinc, J.Ha, S. McLaughlin, J.Barros, and B. Kwak, "LDPC codes for the Gaussian wiretap channel," in Proc. Information Theory Workshop, October 2009.

[7] S. Y. El Rouayheb and E. Soljanin, "On wiretap networks II," in Proceedings ISIT, 2007.

[8] X. He and A. Yener, "Providing secrecy with structured codes: Tools and applications to two-user Gaussian channels," July 2009. [Online]. Available: http://arxiv.org/pdf/0907.5388

[9] A. Wyner, "The wire-tap channel," Bell. Syst. Tech. Journal, vol. 54 October 1975.

[10] J. Conway and N. Sloane, Sphere packings, Lattices and Groups, 3rd ed. Springer-Verlag, 1998.

[11] E. Weisstein, "Jacobi Theta Functions," MathWorld - A Wolfram Web Resource. [Online]. Available: http://mathworld.wolfram.com/JacobiThetaFunctions.html

[12] N.-P. Skoruppa, "Reduction mod $\ell$ of Theta Series of level $\ell^{n}$." [Online]. Available: arXiv:0807.4694v2[math.NT]

[13] W. Ebeling, Lattices and Codes. Advanced Lectures in Mathematics, 1994.

[14] J.-P. Serre, A course in arithmetic, ser. Graduate Texts in Mathematics. Springer, 1996.

[15] C. Bachoc and G. Nebe, "Extremal lattices of minimum 8 related to the Mathieu group $M_{22}$," Journal für die reine und angewandte Mathematik, vol. 494, pp. 155-171, 1998.

[16] H.-G. Quebbemann, "Modular lattices in Euclidean spaces," J. Number Theory, vol. 54, pp. 190-202, 1995. 